\title{
Antibodies to oxidized insulin improve prediction of type 1 diabetes in children with positive standard islet autoantibodies
}

\author{
Rocky Strollo ${ }^{1}$ (D) | Chiara Vinci ${ }^{1,2}$ | Nicola Napoli ${ }^{3}$ (D) | Elvira Fioriti ${ }^{1}$ | \\ Ernesto Maddaloni ${ }^{1}$ (D) । Linda Åkerman ${ }^{5}$ (D) | Rosaura Casas ${ }^{5}$ | Paolo Pozzilli ${ }^{1,4}$ (D) । \\ Johnny Ludvigsson $^{5,6}$ | Ahuva Nissim ${ }^{2}$
}

${ }^{1}$ Department of Medicine, Unit of Endocrinology \& Diabetes, Universitá Campus Bio-Medico di Roma, Rome, Italy

${ }^{2}$ Centre for Biochemical Pharmacology, William Harvey Research Institute, Barts and The London School of Medicine and Dentistry, Queen Mary University of London, London, UK

${ }^{3}$ I.R.C.C.S. Istituto Ortopedico Galeazzi, Milan, Italy

${ }^{4}$ Centre for Immunobiology, the Blizard Institute, Barts and The London School of Medicine and Dentistry, Queen Mary

University of London, London, UK

${ }^{5}$ Division of Pediatrics, Department of Clinical Experimental Medicine, Medical Faculty, Linköping University, Linköping, Sweden

${ }^{6}$ Crown Princess Victoria Children's Hospital, Region Östergötland, Linköping, Sweden

\section{Correspondence}

Rocky Strollo and Paolo Pozzilli, Endocrinology \& Diabetes, Universitá Campus Bio-Medico di Roma, Via Alvaro del Portillo 21, 00128 Rome, Italy.

Email: r.strollo@unicampus.it:

p.pozzilli@unicampus.it

Ahuva Nissim, Centre for Biochemical Pharmacology, William Harvey Research Institute, Barts and The London School of Medicine and Dentistry, Queen Mary University of London, Charterhouse Square, London EC1M 6BQ, UK.

Email: a.nissim@qmul.ac.uk

\begin{abstract}
Background: Antibodies to posttranslationally modified insulin (oxPTM-INS-Ab) are a novel biomarker of type 1 diabetes (T1D). Here, we evaluated whether oxPTM-INS$\mathrm{Ab}$ can improve T1D prediction in children with positive standard islet autoantibodies (AAB).
\end{abstract}

Methods: We evaluated sensitivity, specificity, accuracy, and risk for progression to T1D associated with oxPTM-INS-Ab and the standard islet AAB that include insulin (IAA), GAD (GADA), and tyrosine phosphatase $2(I A-2 A)$ in a cohort of islet AABpositive $\left(\mathrm{AAB}^{+}\right)$children from the general population (median follow-up 8.8 years).

Results: oxPTM-INS-Ab was the most sensitive and specific autoantibody biomarker (74\% sensitivity, 91\% specificity), followed by IA-2A (71\% sensitivity, 91\% specificity). GADA and IAA showed lower sensitivity (65\% and 50\%, respectively) and specificity (66\% and $68 \%$, respectively). Accuracy (AUC of ROC) of oxPTM-INSAb was higher than GADA and IAA ( $P=0.003$ and $P=0.017$, respectively), and similar to IA-2A $(P=0.896)$. oxPTM-INS-Ab and IA-2A were more effective than IAA for detecting progr-T1D when used as second-line biomarker in $\mathrm{GADA}^{+}$children. Risk for diabetes was higher $(P=0.03)$ among multiple $A A B^{+}$who were also oxPTMINS- $\mathrm{Ab}^{+}$compared with those who were oxPTM-INS-A ${ }^{-}$. Importantly, when replacing IAA with oxPTM-INS-Ab, diabetes risk increased to $100 \%$ in children with oxPTMINS- $-\mathrm{Ab}^{+}$in combination with GADA ${ }^{+}$and $I A-2 A^{+}$, compared with $84.37 \%$ in those with $\mathrm{IAA}^{+}, \mathrm{GADA}^{+}$, and IA-2A+ $(P=0.04)$.

Conclusions: Antibodies to oxidized insulin (oxPTM-INS-Ab), compared with IAA which measure autoantibodies to native insulin, improve T1D risk assessment and prediction accuracy in $\mathrm{AAB}^{+}$children.

\section{KEYWORDS}

biomarker, insulin autoantibodies, posttranslational modifications, prediction, type 1 diabetes

Funding information: JDRF, Grant/Award Numbers: 1-SRA-2017-512-Q-R and INO-2015-78-S-B; Swedish Council for Working Life and Social Research, Grant/ Award Number: FAS2004-1775; Medical Research Council of Southeast Sweden (FORSS); JDRF Wallenberg Foundation, Grant/Award Number: K 98-99D-1281301A; Swedish Child Diabetes Foundation; Swedish Research Council, Grant/Award Numbers: K2008-69X-20826-01-4 and K2005-72X-11242-11A; EFSD/JDRF/ Lilly European Programme in Type 1 Diabetes Research, Grant/Award Number: 3-PAR-2016-277-A-N

Rocky Strollo and Chiara Vinci contributed equally to the work. Paolo Pozzilli, Johnny Ludvigsson, and Ahuva Nissim share senior authorship. 


\section{1 | INTRODUCTION}

Type 1 diabetes (T1D) is characterized by a prodromal phase of variable duration marked by islet cytoplasmic autoantibodies (ICA) and circulating islet autoantibodies $(A A B)$ to insulin (IAA), GAD (GADA), tyrosine phosphatase $2(\mathrm{IA}-2 \mathrm{~A})$, and zinc transporter 8 (ZnT8A).

Several approaches have been tested for effective prediction of T1D, ranging from genetic risk assessment, number and levels of islet $A A B$, and appearance of dysglycaemia. ${ }^{1-3}$ Islet $A A B$ represent the most robust approach to predict progression to clinical T1D. ${ }^{4}$ Number of $A A B$ may incrementally increase the risk of progression. ${ }^{1,4}$ When used in combination, islet $A A B$ can identify up to $80 \%$ or more of patients at disease onset or at risk of developing disease, ${ }^{5}$ but except for IA-2A, their predictive potential is extremely reduced when assessed in single test. ${ }^{6}$ Based on this, staging of preclinical T1D has been recently updated to include multiple $A A B$, also termed islet autoimmunity, as the earliest stage of T1D. ${ }^{7}$ Measurement of islet AAB has been instrumental for selecting patients entering clinical trials, but currently fall below the high sensitivity and specificity needed for detection of preclinical diabetes in the general population, where the prevalence of diabetes is of the order of $0.3 \% .^{8}$ Furthermore, newly identified islet antibody specificities might redefine diabetes risk and disease staging.

Recent evidence by our group and others have shown that chemical or enzymatic posttranslational modifications (PTM) of selfantigens, such as oxidation, ${ }^{9,10}$ glycosylation, ${ }^{9}$ citrullination, ${ }^{11,12}$ and deamination, ${ }^{13}$ provide a large and heterogeneous source of neoepitopes able to breach immune tolerance in T1D. This concept is well established in other autoimmune diseases such as rheumatoid arthritis (RA) where citrullination and oxidation are prevalent traits of the disease. Indeed, anticitrullinated peptide antibodies (ACPA) have been included in the classification criteria for RA, ${ }^{14}$ while antibodies to oxidative PTM (oxPTM) collagen type II have been recently proposed as a highly accurate biomarker of the disease. ${ }^{15}$

We have demonstrated the proof of concept that oxPTM of insulin are neoantigens. Most of T1D subjects ${ }^{10}$ or prediabetic children ${ }^{16}$ have autoantibodies to oxPTM insulin (oxPTM-INS-Ab). We showed that oxPTM-INS-Ab is highly accurate for diabetes diagnosis ${ }^{10}$ and may identify subjects who are negative to the other standard islet $\mathrm{AAB}$, and its appearance can antedate diabetes diagnosis by several years. ${ }^{16}$ However, it is not clear whether oxPTM-INS-Ab can improve early diagnosis and prediction in association with the standard islet $A A B$.

Therefore, the primary aim of this study was to evaluate the ability of oxPTM-INS-Ab to predict T1D in children with positive standard islet $A A B$; primary outcome was to evaluate its predictive accuracy compared with autoantibodies to native insulin, IAA. As secondary aim, we evaluated whether oxPTM-INS-Ab was able to refine diabetes risk in multiple $A A B$ positive $\left(A A B^{+}\right)$children. We therefore analysed data available ${ }^{16}$ from $A A B^{+}$children and prediabetic children from the All Babies in Southeast Sweden (ABIS) cohort, a large prospective study in the general population.

\section{2 | MATERIALS AND METHODS}

\section{1 | Subjects}

We used serum samples obtained from the ABIS study, a large prospective birth cohort study in which 17055 unselected children from the general population born between 1 October 1997 to 1 October 1999 have been followed prospectively with regular evaluation of islet AAB before the development of T1D. ${ }^{17,18}$ Of the screened children, 116 developed type 1 diabetes during the follow-up. In the present study, we analysed data available from 23 children progressing to type 1 diabetes (progr-T1D), collected longitudinally before diagnosis at three different time points (at the ages of 5,7 , and 11 years), and 32 children who did not progress to T1D despite being islet $A A B$ positive $\left(\mathrm{NP}-\mathrm{AAB} \mathrm{B}^{+}\right.$), after a median follow-up of 10.8 years (IQR 7.7-12.8). NP$A A B^{+}$children were defined as positive to at least one standard islet AAB (IAA, GADA, and/or IA-2A). Samples were selected where sufficient serum and autoantibody data were available for this study. A comprehensive description of clinical and biochemical features, as well as prevalence of oxPTM-INS-Ab, in the studied population is extensively reported elsewhere. ${ }^{16}$ Progr-T1D were selected irrespective of $A A B$ status; 19 of 23 progr-T1D were positive to at least one standard islet $A A B$ (GADA, IAA, and/or IA-2A), while two were negative to the standard islet $A A B$ but positive to oxPTM-INS-Ab. Overall, progr-T1D and $\mathrm{NP}-\mathrm{AAB}^{+}$were all positive to at least one of the following tested autoantibodies-GADA, IAA, IA-2A, and oxPTM-INS-Ab. Informed consent was obtained from parents prior to the collection of blood. The study was approved by the Research Ethics Committees of the Medical Faculties of Linköping University, Linköping, and Lund University, Lund, Sweden, and by the Ethics Committee of the Universitá Campus Bio-Medico di Roma.

\section{2 | Detection of oxPTM-INS-Ab}

Antibodies to insulin modified by hydroxyl radical (oxPTM-INS) were measured by our developed ELISA platform. Insulin was chemically modified as previously described to generate oxPTM-INS modified by hydroxyl radical $\left({ }^{\circ} \mathrm{OH}\right) .{ }^{10,16}$ Hen egg lysozyme (HEL; Sigma) was similarly modified and used as control antigens. An ELISA was performed using native and oxPTM-INS or control native and oxPTMHEL as targets. Development and calibration of the ELISA are described in our previous publications. ${ }^{10,16}$ Briefly, ELISA plates (Nunc, London, UK) were coated with $10 \mathrm{\mu g} / \mathrm{ml}$ of modified or native protein in $0.05 \mathrm{~mol} / \mathrm{l}$ carbonate/bicarbonate buffer $(\mathrm{pH} 9.6)$ at $4^{\circ} \mathrm{C}$ overnight. Plates were then washed three times with PBS. After blocking for 2 hours with 5\% BSA in 0.5\% Tween PBS, $100 \mu \mathrm{l}$ of 1:200-diluted serum samples in $5 \%$ BSA in $0.5 \%$ Tween PBS were added to each well, followed by 2-hour incubation at room temperature. Plates were then washed with PBS plus 0.1\% Tween, followed by three washes with PBS. Anti-human IgG-horseradish peroxidase-conjugated antibodies (Sigma-Aldrich) were then added at 1:1000 dilution in 5\% BSA in $0.5 \%$ Tween PBS for another 2-hour incubation. The ELISA 


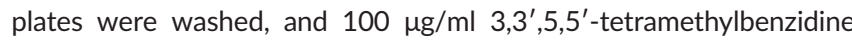
substrate (Sigma-Aldrich) in $100 \mathrm{mmol} / \mathrm{l}$ sodium acetate $(\mathrm{pH}$ 6.0) was added. Subsequently, the reaction was stopped with $1 \mathrm{~mol} / \mathrm{l}$ sulfuric acid. The absorbance was measured at $450 \mathrm{~nm}$ using a GENios plate reader and Magellan software (Tecan, Reading, UK). The ELISA absorbance values obtained for HEL and oxPTM-HEL were used as background controls that were subtracted from the absorbance values of NT-INS and oxPTM-INS, respectively. Levels of oxPTM-INS-Ab above the 99th percentile of 88 healthy individuals were defined as ELISA cut-off. Intra-assay CV was $<8 \%(n=10)$. Interassay CVs were $<10 \%$ and $<13 \%$ for NT-INS and oxPTM-INS-Ab $(n=12)$, respectively.

\section{3 | Standard islet AAB determinations}

The standard islet $A A B$ were measured by radiobinding assay (RBA) as previously described. ${ }^{16,18,19}$ In the 2005 Diabetes Autoantibody Standardization Programme (DASP), the RBA assays for GADA, IA-2A, and IAA achieved $76 \%, 72 \%$, and $28 \%$ sensitivity with $96 \%, 100 \%$, and $100 \%$ specificity, respectively.

\section{4 | Sample size calculation}

Sample size was calculated post hoc based on the results generated in our previous publication ${ }^{16}$ to determine if the number of available sera would suffice for the hypothesis that oxPTM-INS-Ab would be more accurate than at least one standard islet $A A B$ for identifying children progressing to diabetes. Using a significance level of 0.05 and $80 \%$ power, the minimum sample size required was of 23 subjects per group (progr-T1D and NP-AAB ${ }^{+}$). This was based on the area under curve (AUC) of ROC for oxPTM-INS-Ab and GADA of 0.86 and 0.56 , respectively, and on the rank correlation coefficient between oxPTM-INS-Ab and GADA of -0.05 and -0.21 in progr-T1D and $\mathrm{NP}-\mathrm{AAB}^{+}$groups, respectively.

\section{5 | Statistical analysis}

Statistical analyses were performed using Prism Software version 6.01 (GraphPad, San Diego, CA, USA) and MedCalc Statistical Software version 16.8.4 (MedCalc Software bvba, Ostend, Belgium). Diagnostic ability of oxPTM-INS-Ab vs the standard islet $A A B$ was evaluated by receiver operating characteristic (ROC) curve analysis. ROC curves displaying sensitivity and specificity of a continuous marker (titre or absorbance) when comparing two markers, or a binary disease variable (positive vs negative) when comparing combinations of multiple markers, were tested. To evaluate sensitivity and specificity of continuous markers (titre or absorbance), cut-off points of positivity were specifically recalculated for the population studied based on the Youden's index $J$ obtained by the ROC analysis. Area under the curve (AUC) of the ROC (ROC-AUC) of OxPTM-INS-Ab and the standard islet $A A B$ is reported as absolute value \pm standard error and was tested for equality according to DeLong et al. ${ }^{20}$ The overall accuracy (\%) was calculated as (number of progr-T1D identified as positive + number of NP-AAB ${ }^{+}$identified as negative) / (total number of progr-T1D + total number of NP-AAB ${ }^{+}$. This calculation was based on predetermined cut-offs obtained from healthy individuals as reported in Section 2 and as described previously. ${ }^{18,19}$ Categorical variables between groups were compared by the Fisher exact test. Survival analysis for diabetes onset used the Kaplan-Meier method. The log-rank test was used to compare cumulative incidence of diabetes between groups. The Wilcoxon test was used when proportional hazard validity was not respected. This study had only one primary aim. The primary outcome of this aim was the difference in the ROCAUC between ox-PTM-INS-Ab and IAA, which was explored with one single test of equality (according to DeLong et al). Therefore, no correction for multiple testing was performed.

\section{3 | RESULTS}

\section{1 | Prevalence of oxPTM-INS-Ab and the standard $A A B$}

Prevalence of oxPTM-INS-Ab and the standard AAB is summarized in Table 1. A detailed description of the distribution and overlapping prevalence of oxPTM-INS-Ab and the standard islet AAB in the tested population has been reported elsewhere. ${ }^{16}$ Prevalence of oxPTM-INS$\mathrm{Ab}$ was higher in progr-T1D compared with the NP-AAB ${ }^{+}[82 \%(19 /$ 23) vs $19 \%(6 / 32), P<0.0001$ ]. In contrast, prevalence of GADA and IAA did not differ significantly between progr-T1D and NP-AAB ${ }^{+}$ groups [GADA: $78 \%(18 / 23)$ vs $91 \%(29 / 32), P=0.257$; IAA: $56 \%$ (10/18) vs $33 \%(9 / 27), P=0.218]$, while IA-2A were more prevalent in the progr-T1D group [70\% (16/23) vs 13\% (4/32), $P<0.0001]$.

\subsection{Sensitivity, specificity, and accuracy indices of oxPTM-INS-Ab and the standard AAB}

ROC analyses were performed to obtain sensitivity, specificity, and accuracy (AUC) of oxPTM-INS-Ab in comparison with the standard autoantibodies for discriminating progr-T1D from NP-AAB ${ }^{+}$children. Best cut-off points for this high-risk population were derived from the Youden's index $J$ of each biomarker. Among the four tested biomarkers, oxPTM-INS-Ab and IA-2A displayed the best sensitivity and specificity; oxPTM-INS-Ab had 74\% sensitivity and $91 \%$ specificity, while IA-2A had $71 \%$ sensitivity and $91 \%$ specificity, respectively. Conversely, GADA and IAA showed lower sensitivity (65\% and 50\%, respectively) and specificity (66\% and $68 \%$, respectively) (Table 2 ). The ROC-derived accuracy (AUC) of oxPTM-INS-Ab for identification of progr-T1D was higher than GADA (AUC $0.862 \pm 0.058$ vs $0.566 \pm 0.093 ; P=0.003$ ) and IAA (AUC $0.862 \pm 0.058$ vs $0.624 \pm 0.082 ; P=0.017$ ) and similar to IA-2A (AUC $0.862 \pm 0.058$ vs $0.871 \pm 0.049 ; P=n s)$. Similarly, the overall accuracy, based on predetermined cut-off points, was higher for oxPTM-INS (82\%) and IA-2A (80\%), followed by IAA (62\%) and GADA (38\%), respectively (Table 2). 
TABLE 1 Prevalence of oxPTM-INS-Ab and the standard islet autoantibodies

\begin{tabular}{lccc} 
& AAB $^{+}$Children & Progr-T1D & NP-AAB ${ }^{+}$ \\
\hline $\mathrm{N}$ & 55 & 23 & 32 \\
\hline $\begin{array}{l}\text { Follow-up time, } \\
\text { years }\end{array}$ & $\begin{array}{c}8.75(5.96, \\
10.78)\end{array}$ & $\begin{array}{c}5.12(3.22, \\
7.73)\end{array}$ & $\begin{array}{c}10.78(7.68, \\
12.85)\end{array}$ \\
\hline GADA & $47(85 \%)$ & $18(78 \%)$ & $29(91 \%)$ \\
\hline IAA $^{\mathrm{a}}$ & $19(42 \%)$ & $10(56 \%)$ & $9(33 \%)$ \\
\hline IA-2A $^{\text {oxPTM-INS-Ab }}$ & $20(36 \%)$ & $16(70 \%)$ & $4(13 \%)$ \\
\hline GADA only & $25(45 \%)$ & $19(82 \%)$ & $6(19 \%)$ \\
\hline IAA only & $23(42 \%)$ & $1(4 \%)$ & $22(69 \%)$ \\
\hline $\begin{array}{l}\text { IA-2A only } \\
\text { oxPTM-INS-Ab } \\
\text { only }\end{array}$ & $2(4 \%)$ & $1(6 \%)$ & $1(4 \%)$ \\
\hline $\begin{array}{l}\text { Multiple standard } \\
\text { AAB }\end{array}$ & $26(47 \%)$ & $18(78 \%)$ & $8(25 \%)$ \\
\hline
\end{tabular}

Follow-up time is reported as median (interquartile range).

aAA was available in 45 children (18 progr-T1D and 27 NP-AAB ${ }^{+}$).

\subsection{Evaluation of oxPTM-INS-Ab for prediction of T1D in GADA-positive children}

To evaluate the efficiency of oxPTM-INS-Ab as a second-line Ab for the prediction of T1D, we compared the ROC-AUC of oxPTM-INS$A b$ with those of IAA and IA-2A in subjects positive for GADA at the cut-off point used in the general population. In this group, both IA-2A and oxPTM-INS-Ab were highly accurate (AUC of IA$2 \mathrm{~A}=0.941 \pm 0.04$ vs $\mathrm{AUC}$ of oxPTM-INS-Ab $=0.911 \pm 0.05$; $P=0.620)$ and more effective than IAA for detecting progr-T1D (AUC-IAA $=0.679 \pm 0.09 ; P=0.024$ vs oxPTM-INS-Ab and $P=0.009$ vs $(\mathrm{A}-2 \mathrm{~A})$. When two more autoantibodies were added to GADA, we found that IA-2A/oxPTM-INS-Ab provided the best combination with the highest accuracy (AUC $=0.960$ ), which was superior to the IA-2A/IAA or oxPTM-INS-Ab/IAA $(P=0.002)$.

TABLE 2 Comparison of the performance characteristics of oxPTMINS-Ab and the standard islet autoantibodies

\begin{tabular}{|c|c|c|c|c|c|}
\hline Antibody & $\begin{array}{l}\% \\
\text { Sensitivity }\end{array}$ & $\begin{array}{l}\% \\
\text { Specificity }\end{array}$ & $\mathrm{AUC} \pm \mathrm{SE}$ & $\begin{array}{l}\% \\
\text { Accuracy }\end{array}$ & $\begin{array}{l}P \text { vs } \\
\text { oxPTM- } \\
\text { INS-Ab }\end{array}$ \\
\hline $\begin{array}{l}\text { oxPTM- } \\
\text { INS- } \\
\text { Ab }\end{array}$ & 74 & 91 & $0.862 \pm 0.058$ & 81.8 & NA \\
\hline GADA & 65 & 66 & $0.566 \pm 0.093$ & 38.2 & 0.003 \\
\hline IAA & 50 & 68 & $0.624 \pm 0.082$ & 62.2 & 0.017 \\
\hline $\mid A-2 A$ & 71 & 91 & $0.871 \pm 0.049$ & 80.0 & 0.896 \\
\hline
\end{tabular}

Sensitivity and specificity are derived from the ROC analysis based on the Youden's index $J$ of each biomarker; \% accuracy is calculated as (number of progr-T1D identified as positive + number of NP-AAB ${ }^{+}$identified as negative) / (total number of progr-T1D + total number of NP-AAB ${ }^{+}$), based on data reported in Table 1. $P$ value refers to differences of AUC between oxPTM-INS-Ab and the standard islet autoantibodies.

\subsection{Risk of progression to clinical diabetes in children positive to at least one standard autoantibody}

The risk for diabetes increased incrementally according to the number of positive tests for the standard AAB (GADA, IA-2A, and IAA). Thus, the 10 -year risk (95\% confidence limits) in children positive for one, two, and three autoantibodies were 6\% (1\%-67\%), 70\% (47\%-84\%), and $84 \%$ (75\%-94\%), respectively. A total of $18 / 23$ children (78\%) with multiple standard $A A B$ progressed to diabetes (HR compared with single islet $A A B, 23.8$ [95\% $\mathrm{Cl}, 3.6-22.5]$ ) after a median followup of 7.47 years (Figure 1A). oxPTM-INS-Ab was able to stratify multiple $\mathrm{AAB}^{+}$children in two subgroups with different risk. Figure $1 \mathrm{~B}$ shows diabetes risks in children positive for at least two standard $A A B$, subdivided by oxPTM-INS-Ab. The risk for diabetes was significantly higher $(P=0.03)$ among multiple (two or more) $\mathrm{AAB}^{+}(\mathrm{IAA}, \mathrm{IA}-$ 2A, GAD) who were also oxPTM-INS-Ab ${ }^{+}$compared with those who were oxPTM-INS-Ab- In these two groups, the 10-year cumulative diabetes incidences (95\% confidence limits) were $87 \%$ (77-93) and $56 \%$ (14-84), respectively. Importantly, by replacing IAA with oxPTM-INS-Ab, children that were GADA, IA-2A, and oxPTM-INS

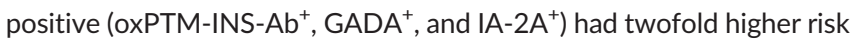
of progression to clinical diabetes within 5 years $(66.67 \%[95 \% \mathrm{Cl}$ 43.17-85.25]) compared with children with the three standard $A A B$ (25\% [95\% Cl 1-70.82\%]). At 10 years of follow-up, diabetes risk increased to $100 \%$ in children with oxPTM-INS-Ab, GADA, and IA$2 \mathrm{~A}$, compared with $84.37 \%(95 \% \mathrm{Cl} 64.18-93.69 ; P=0.04)$ in those with IAA, GADA, and IA-2A (Figure 1C).

\section{4 | DISCUSSION}

Our results suggest that the inclusion of oxPTM-INS-Ab in the panel of autoantibody assay was able to improve significantly the prediction of T1D. We found that oxPTM-INS-Ab positivity increased risk in multiple $A A B^{+}$children. oxPTM-INS-Ab and IA-2A were more effective than IAA for detecting children progressing to T1D when used as second-line marker in GADA-positive children.

In this study, oxPTM-INS showed better accuracy (AUC of ROC) than IAA and GADA. The superiority of oxPTM-INS-Ab over IAA is consistent with a previous study carried out by our group. In newly diagnosed T1D, oxPTM-INS-Ab was more sensitive than IAA (84\% vs $61 \%$ ), detecting over one-third of subjects negative to IAA. ${ }^{10} \mathrm{~A}$ similar result was obtained in the ABIS cohort, where oxPTM-INS-Ab identified $38 \%$ of prediabetic children who were negative to IAA. ${ }^{16}$ More recently, Sidney et al showed that peptides derived from oxPTM-INS B-chain can bind HLA class I with better affinity than unmodified insulin peptides. ${ }^{21}$ Taken together, such findings fit with the hypothesis that insulin autoimmunity is predominant in most T1D subjects and that oxidative modifications may amplify (and theoretically trigger) autoimmune reactions against insulin. Circulating markers of oxidative stress are increased not only in diabetes ${ }^{22}$ but also in euglycaemic subjects at risk for developing the disease. ${ }^{23} \mathrm{We}$ speculate that such early changes in redox status may lead to insulin 
A

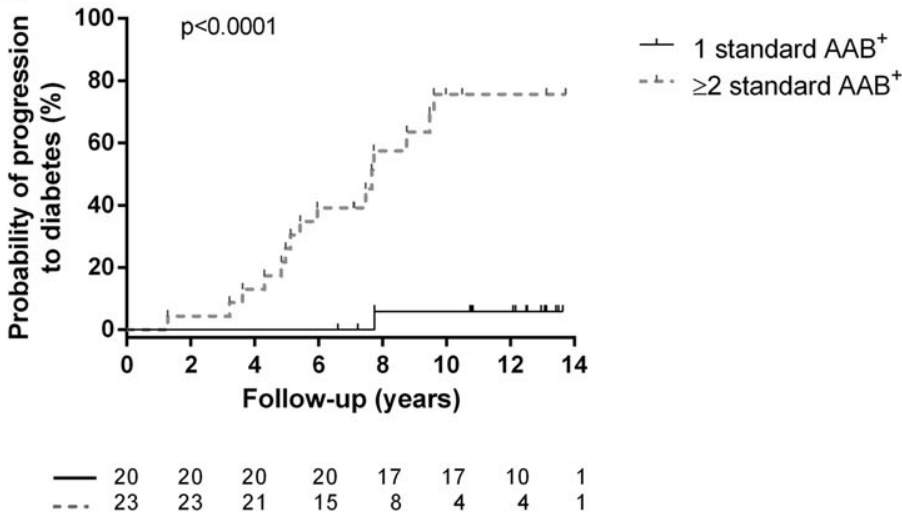

B

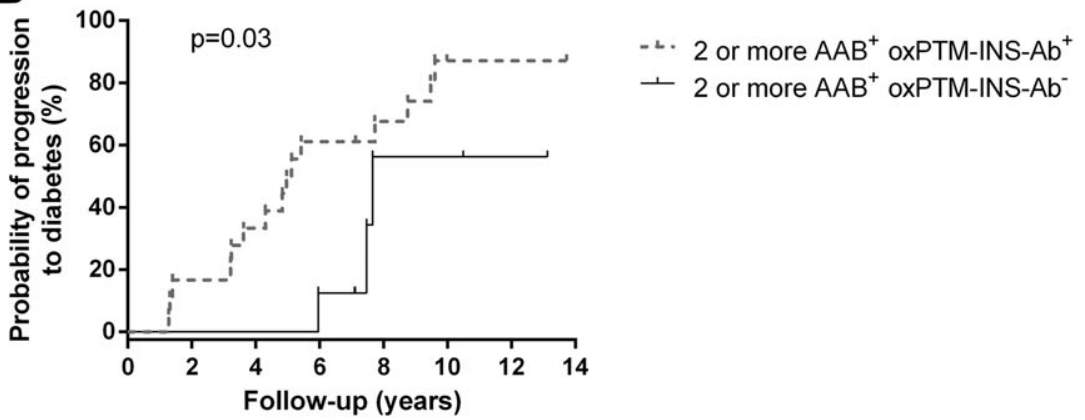

$\begin{array}{rrrrrrrrr} & 8 & 8 & 8 & 8 & 3 & 3 & 2 & 1 \\ --. & 18 & 16 & 13 & 8 & 8 & 6 & 2 & 1\end{array}$

FIGURE 1 Probability of progression to diabetes in $\mathrm{AAB}^{+}$children from the $\mathrm{ABIS}$ study. A, Probability of progression to diabetes according to the number of standard $\mathrm{AAB}^{+}$tests for GADA, IAA, and IA-2A. Risk of progression was higher in children with multiple vs single positive AAB $(P<0.0001)$. B, Probability of progression in multiple standard $\mathrm{AAB}^{+}$children, according to oxPTM-INS-Ab status. oxPTM-INS-A $b^{+}$subjects had higher risk than those who were oxPTM-INS-Ab$(P=0.03$, Wilcoxon test). C, Probability of progression in triple $\mathrm{AAB}^{+}$increased when oxPTM-INS-Ab was used in place of IAA $(P=0.04)$. Number of subjects at risk is showed below each graph

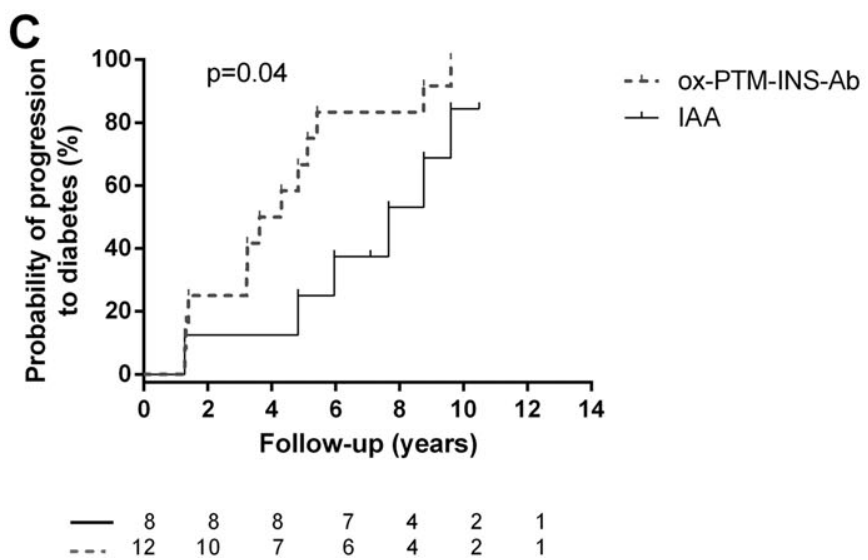

modifications and then autoimmunity in those who are immunologically susceptible to recognize such modifications as abnormal or unable to clear the modified antigen. We hypothesize two main mechanisms for the involvement of insulin modification in T1D immune pathogenesis: (1) generation of oxPTM by reactive oxidants released by leukocytes during insulitis or (2) by altered redox state within beta-cell under stress. The concept that posttranslational modifications of insulin may enhance autoimmune reactions in diabetes is not limited to oxPTM. Other modifications of (pro)insulin, such as deamidation of C-peptide ${ }^{13}$ and covalent cross-linking of proinsulin peptides to other peptides (insulin hybrid peptides), ${ }^{24}$ are targets of $\mathrm{T}$ cell responses. More recently, Wang et al showed that mutations 
at the C-terminal end of the insulin epitope B9:23 dramatically improved presentation to pathogenic $\mathrm{T}$ cells, suggesting PTM-like transpeptidation as another potential mechanism to create pathogenic epitopes in T1D. ${ }^{25}$

In the present study, replacement of IAA with oxPTM-INS-Ab lead to improved prediction. This can be justified by the low prevalence and low specificity of IAA. This is consistent with results from two other studies carried out in the general populations. In the study by Knip and collaborator, IAA showed low sensitivity being detected only in $28 \%$ (5 of 18) subjects progressing to T1D (age range 2.9 to 18.9 years), ${ }^{26}$ a percentage even lower than in our study (55\%). The low IAA specificity in our study is similar to that from the Washington State Diabetes Prediction Study carried out on school children from the general population; in this study, 39\% (40/102) of children with single positive islet $A A B$ were positive to IAA but did not progress to T1D. ${ }^{27}$ IAA prevalence may vary according to clinical and genetic features of the study populations, being higher in infants and cohorts selected for high genetic or familiar risk. For example, in the DAISY study carried out on young children with increased genetic susceptibility to T1D, 22 of 24 children developing diabetes were IAA positive (age at diagnosis 1 to 3 years). ${ }^{28}$ Similar prevalence for IAA was found in progressor children from the DIPP ${ }^{29}$ and the BABY-DIAB ${ }^{30}$ studies. However, IAA disappear more often than other $A A B,{ }^{29}$ and loosing IAA reactivity is associated with delayed progression to T1D in multiple AAB-positive children. ${ }^{31,32}$ Therefore, we cannot exclude that children defined as IAA negative in our study have not been previously IAA positive.

An interesting finding of our study is the low accuracy of GADA. This is not completely unexpected, as GADA is less effective in predicting diabetes than other islet AAB. In a study on genetically atrisk subjects from three large prospective cohorts, the 10-year risk of progression conferred by GADA was about threefold lower than that provided by IA-2A (12.9\% vs $40.5 \%) .{ }^{4}$ This is also in line with the study by Yu et al performed in T1D relatives, where GADA failed to independently predict diabetes progression over a 5-year followup. ${ }^{33}$ In the same study, the risk associated with GADA was up to fourfold lower that that conferred by IA-2A or IAA. ${ }^{33}$ In a recent cluster analysis of the TEDDY study, stable single GADA positivity (with or without IAA) conferred $30 \%$ or lower diabetogenic effect than IA-2A clusters. ${ }^{31}$ However, these results should be interpreted with caution. Features specific to our population, including the high prevalence of GADA (85\%) and its tendency to be the most prevalent single autoantibody, may also explain our finding. This is consistent with studies in T1D relatives. GADA was highly prevalent $(74 \%)$ in first-, second-, or third-degree T1D relatives from the TrialNet cohort ${ }^{33}$ and the most prevalent autoantibody in initially single positive relatives from the Belgian Diabetes Registry. ${ }^{34}$ However, we cannot exclude that subjects positive to GADA will develop diabetes at a later follow-up not yet assessed in our study. According to the report from the Belgian Diabetes Registry, relatives positive to GADA and IAA progressed more slowly than double autoantibody-positive individuals carrying IA-2A and/or ZnT8A. ${ }^{34}$ In a study by Knip and collaborators on subjects from the general population, 6 of 18 subjects (33\%) developed diabetes after age of 20 ( 2 of 18 after age of 30 ), despite being islet autoantibody positive for up to 20 years. ${ }^{26}$ It is also worth noting that GADA as single antibody is the most common antibody biomarker for adult onset autoimmune diabetes. ${ }^{35}$

As reported by others, we also found an association between number of positive standard autoantibodies and risk of progression to diabetes. ${ }^{1,4,26,33,34}$ In the group of multiple autoantibody positive, oxPTM-INS-Ab testing refined risk by identifying a subgroup with a faster and greater risk of progression. This is similar to what has been shown for ZnT8A in a study on T1D relatives from the TrialNet cohort. ${ }^{33}$ Therefore, these data may have implications for disease prediction and staging. Islet autoimmunity (as defined by the presence of two or more islet $A A B$ ) represents the earliest stage of T1D and identifies a target population for prevention trials and future preventive strategies. ${ }^{7}$ Therefore, improved identification of subjects progressing to clinical disease has important clinical implications. If confirmed in larger studies, oxPTM-INS-Ab-based assay may be implemented as additional biomarker to further redefine disease taxonomy, allowing better prediction and therefore better stratification into T1D prevention trials.

The main strength of our study is the evaluation of oxPTM-INS$\mathrm{Ab}$ in a well-characterized cohort of children from the general population, tested for the standard diabetes $A A B$ and the fact that replacing IAA with oxPTM-INS-Ab in the equation resulted in a better prediction of risk to develop T1D. This discovery needs to be confirmed using independent and larger cohorts. Our study has limitations. First, the number of children progressing to T1D with a single standard autoantibody was small, and this did not allow us to find out whether oxPTM-INS-Ab would significantly redefine the risk in this category of subjects. Second, it was not possible to measure oxPTM-INS-Ab before seroconversion of the other islet $A A B$; thus, we cannot infer about timing of oxPTM-INS-Ab appearance compared with the other biomarkers. Third, although oxPTM-INS-Ab were not present in children with negative standard islet $A A B$, this should be further confirmed in larger cohorts of low-risk individuals. Finally, the post hoc sample size calculation is a limitation of the study. Although our study was powered to address the general question of the performance of oxPTM-INS-Ab against any other islet $A A B$, such data are based on minimal differences in the absolute number of subjects.

In conclusion, oxPTM-INS-Ab may identify children progressing to T1D with better accuracy than IAA and in combination with the current standard autoantibody markers such as GADA and IA-2A. Thus, oxPTM-INS-Ab may refine risk in multiple autoantibody-positive children and may maximize diabetes risk progression in children positive to the other islet $A A B$ biomarkers. Additional studies with larger cohorts are required to confirm the predictive potential of oxPTMINS-Ab in T1D.

\section{FUNDING}

This study was supported by the EFSD/JDRF/Lilly European Programme in Type 1 Diabetes Research 3-PAR-2016-277-A-N (to RS, AN, PP), by JDRF grants INO-2015-78-S-B (to PP, AN, RS) and JDRF 1-SRA-2017-512-Q-R (to AN, RS, PP, JL). RS was in part supported by 
the EFSD Future Leaders Mentorship Programme. ABIS and the autoantibody determinations have been supported by the Swedish Research Council (K2005-72X-11242-11A and K2008-69X-2082601-4), the Swedish Child Diabetes Foundation (Barndiabetesfonden), JDRF Wallenberg Foundation (K 98-99D-12813-01A), Medical Research Council of Southeast Sweden (FORSS), and the Swedish Council for Working Life and Social Research (FAS2004-1775).

\section{DISCLOSURE STATEMENT}

The authors have nothing to disclose.

\section{CONTRIBUTION STATEMENT}

RS, PP, JL, and AN conceived the study. JL is the project leader of the ABIS study. CV performed experiments and provided the data. NN, $\mathrm{EF}$, and EM contributed to data acquisition and analysis. LA and RC contributed to the islet autoantibody determination. RS analysed the data and wrote the first draft. All authors critically revised the manuscript for intellectual content. All authors have seen and approved the final draft. RS is the guarantor of this work and, as such, had full access to all the data in the study and takes responsibility for the integrity of the data and the accuracy of the data analysis.

\section{ABBREVIATIONS}

$\begin{array}{ll}\text { AAB } & \text { autoantibody } \\ \text { AAB }^{+} & \text {autoantibody positive } \\ \text { ABIS } & \text { All Babies in Southeast Sweden } \\ \text { ACPA } & \text { anticitrullinated peptides antibody } \\ \text { AUC } & \text { area under the curve } \\ \text { BSA } & \text { bovine serum albumin } \\ \text { ELISA } & \text { enzyme-linked immunosorbent assay } \\ \text { GADA } & \text { GAD autoantibodies } \\ \text { HEL } & \text { hen egg lysozime } \\ \text { IA-2A } & \text { tyrosin phosphatase autoantibody } \\ \text { IAA } & \text { insulin autoantibodies } \\ \text { NP-AAB } & \text { nonprogressors with positive autoantibodies } \\ \text { oxPTM } & \text { oxidative PTM } \\ \text { oxPTM-INS } & \text { oxidatively posttranslationally modified insulin } \\ \text { oxPTM-INS-Ab } & \text { antibody to oxPTM-INS } \\ \text { PTM } & \text { posttranslational modifications } \\ \text { RA } & \text { rheumatoid arthritis } \\ \text { RBA } & \text { radiobinding assay } \\ \text { ROC } & \text { receiver operating characteristic } \\ \text { T1D } & \text { type 1 diabetes } \\ \text { ZnT8A } & \text { zinc transporter 8 autoantibodies } \\ & \end{array}$

\section{ORCID}

Rocky Strollo (1) https://orcid.org/0000-0001-8967-7613

Nicola Napoli (1) https://orcid.org/0000-0002-3091-8205

Ernesto Maddaloni (D) https://orcid.org/0000-0003-3844-9463

Linda Åkerman (D) https://orcid.org/0000-0003-1504-3655
Paolo Pozzilli (D) https://orcid.org/0000-0001-5090-636X

\section{REFERENCES}

1. Bonifacio E. Predicting type 1 diabetes using biomarkers. Diabetes Care. 2015;38(6):989-996.

2. Vehik K, Cuthbertson D, Boulware D, et al. Performance of HbA1c as an early diagnostic indicator of type 1 diabetes in children and youth. Diabetes Care. 2012;35(9):1821-1825.

3. Sosenko JM, Skyler JS, Palmer JP, et al. The prediction of type 1 diabetes by multiple autoantibody levels and their incorporation into an autoantibody risk score in relatives of type 1 diabetic patients. Diabetes Care. 2013;36(9):2615-2620.

4. Ziegler AG, Rewers M, Simell O, et al. Seroconversion to multiple islet autoantibodies and risk of progression to diabetes in children. Jama. 2013;309(23):2473-2479.

5. Orban T, Sosenko JM, Cuthbertson D, et al. Pancreatic islet autoantibodies as predictors of type 1 diabetes in the Diabetes Prevention Trial-Type 1. Diabetes Care. 2009;32(12):2269-2274.

6. Akerman L, Ludvigsson J, Swartling U, Casas R. Characteristics of the pre-diabetic period in children with high risk of type 1 diabetes recruited from the general Swedish population-the ABIS study. Diabetes Metab Res Rev. 2017;33(6).

7. Insel RA, Dunne JL, Atkinson MA, et al. Staging presymptomatic type 1 diabetes: a scientific statement of JDRF, the Endocrine Society, and the American Diabetes Association. Diabetes Care. 2015;38(10): 1964-1974.

8. Hermann R, Bartsocas CS, Soltész G, et al. Genetic screening for individuals at high risk for type 1 diabetes in the general population using HLA class II alleles as disease markers. A comparison between three European populations with variable rates of disease incidence. Diabetes Metab Res Rev. 2004;20(4):322-329.

9. Strollo R, Rizzo P, Spoletini M, et al. HLA-dependent autoantibodies against post-translationally modified collagen type II in type 1 diabetes mellitus. Diabetologia. 2013;56(3):563-572.

10. Strollo R, Vinci C, Arshad MH, et al. Antibodies to post-translationally modified insulin in type 1 diabetes. Diabetologia. 2015;58(12):2851-2860.

11. Rondas D, Crèvecoeur I, D'Hertog W, et al. Citrullinated glucoseregulated protein 78 is an autoantigen in type 1 diabetes. Diabetes. 2015;64(2):573-586

12. McGinty JW, Chow IT, Greenbaum C, Odegard J, Kwok WW, James EA. Recognition of posttranslationally modified GAD65 epitopes in subjects with type 1 diabetes. Diabetes. 2014;63(9):3033-3040.

13. van Lummel M, Duinkerken G, van Veelen PA, et al. Posttranslational modification of HLA-DQ binding islet autoantigens in type 1 diabetes. Diabetes. 2014;63(1):237-247.

14. Aletaha D, Neogi T, Silman AJ, et al. 2010 Rheumatoid arthritis classification criteria: an American College of Rheumatology/European League Against Rheumatism collaborative initiative. Arthritis Rheum. 2010;62(9):2569-2581.

15. Strollo R, Ponchel F, Malmström V, et al. Autoantibodies to posttranslationally modified type II collagen as potential biomarkers for rheumatoid arthritis. Arthritis Rheum. 2013;65(7):1702-1712.

16. Strollo R, Vinci C, Napoli N, Pozzilli P, Ludvigsson J, Nissim A. Antibodies to post-translationally modified insulin as a novel biomarker for prediction of type 1 diabetes in children. Diabetologia. 2017;60(8):1467-1474.

17. Ludvigsson J, Ludvigsson M, Sepa A. Screening for prediabetes in the general child population: maternal attitude to participation. Pediatr Diabetes. 2001;2(4):170-174. 
18. Wahlberg J, Vaarala O, Ludvigsson J. Asthma and allergic symptoms and type 1 diabetes-related autoantibodies in 2.5-yr-old children. Pediatr Diabetes. 2011;12(7):604-610.

19. Holmberg H, Wahlberg J, Vaarala O, Ludvigsson J. Short duration of breast-feeding as a risk-factor for beta-cell autoantibodies in 5-yearold children from the general population. $\mathrm{Br} J$ Nutr. 2007;97(1):111-116.

20. DeLong ER, DeLong DM, Clarke-Pearson DL. Comparing the areas under two or more correlated receiver operating characteristic curves: a nonparametric approach. Biometrics. 1988;44(3):837-845.

21. Sidney J, Vela JL, Friedrich D, et al. Low HLA binding of diabetesassociated CD8+ T-cell epitopes is increased by post translational modifications. BMC Immunol. 2018;19(1):12.

22. Marra G, Cotroneo P, Pitocco D, et al. Early increase of oxidative stress and reduced antioxidant defenses in patients with uncomplicated type 1 diabetes: a case for gender difference. Diabetes Care. 2002;25(2): 370-375.

23. Matteucci E, Giampietro O. Oxidative stress in families of type 1 diabetic patients. Diabetes Care. 2000;23(8):1182-1186.

24. Delong T, Wiles TA, Baker RL, et al. Pathogenic CD4 T cells in type 1 diabetes recognize epitopes formed by peptide fusion. Science. 2016;351(6274):711-714.

25. Wang Y, Sosinowski T, Novikov A, et al. C-terminal modification of the insulin B:11-23 peptide creates superagonists in mouse and human type 1 diabetes. Proc Natl Acad Sci U S a. 2018;115(1):162-167.

26. Knip $M$, Korhonen S, Kulmala $P$, et al. Prediction of type 1 diabetes in the general population. Diabetes Care. 2010;33(6):1206-1212.

27. LaGasse JM, Brantley MS, Leech NJ, et al. Successful prospective prediction of type 1 diabetes in schoolchildren through multiple defined autoantibodies: an 8-year follow-up of the Washington State Diabetes Prediction Study. Diabetes Care. 2002;25(3):505-511.

28. Barker JM, Barriga KJ, Yu L, et al. Prediction of autoantibody positivity and progression to type 1 diabetes: Diabetes Autoimmunity Study in the Young (DAISY). J Clin Endocrinol Metab. 2004;89(8):3896-3902.
29. Kimpimäki T, Kulmala P, Savola K, et al. Natural history of beta-cell autoimmunity in young children with increased genetic susceptibility to type 1 diabetes recruited from the general population. J Clin Endocrinol Metab. 2002;87(10):4572-4579.

30. Ziegler AG, Hummel M, Schenker M, Bonifacio E. Autoantibody appearance and risk for development of childhood diabetes in offspring of parents with type 1 diabetes: the 2-year analysis of the German BABYDIAB study. Diabetes. 1999;48(3):460-468.

31. Endesfelder D, Zu Castell W, Bonifacio E, et al. Time-resolved autoantibody profiling facilitates stratification of preclinical type 1 diabetes in children. Diabetes. 2019;68(1):119-130.

32. Endesfelder D, Hagen M, Winkler C, et al. A novel approach for the analysis of longitudinal profiles reveals delayed progression to type 1 diabetes in a subgroup of multiple-islet-autoantibody-positive children. Diabetologia. 2016;59(10):2172-2180.

33. Yu L, Boulware DC, Beam CA, et al. Zinc transporter-8 autoantibodies improve prediction of type 1 diabetes in relatives positive for the standard biochemical autoantibodies. Diabetes Care. 2012;35(6): 1213-1218.

34. Gorus FK, Balti EV, Messaaoui A, et al. Twenty-year progression rate to clinical onset according to autoantibody profile, age, and HLA-DQ genotype in a registry-based group of children and adults with a first-degree relative with type 1 diabetes. Diabetes Care. 2017;40(8):1065-1072.

35. Leslie RD, Kolb H, Schloot NC, et al. Diabetes classification: grey zones, sound and smoke: action LADA 1. Diabetes Metab Res Rev. 2008;24(7):511-519.

How to cite this article: Strollo R, Vinci C, Napoli N, et al. Antibodies to oxidized insulin improve prediction of type 1 diabetes in children with positive standard islet autoantibodies. Diabetes Metab Res Rev. 2019;e3132. https://doi.org/ 10.1002/dmrr.3132 